\title{
Exercise Intensity during Treadmill Walking with Gait-Patterned FES among Patients with Incomplete Spinal Cord Injury: Case Series
}

\author{
Masae Miyatani, ${ }^{1}$ Kei Masani, ${ }^{1}$ Noritaka Kawashima, ${ }^{2}$ B. Cathy Craven, ${ }^{1,3}$ \\ T. Adam Thrasher, ${ }^{4}$ and Milos R. Popovic ${ }^{1,5}$ \\ ${ }^{1}$ Lyndhurst Centre, Toronto Rehabilitation Institute, 520 Sutherland Drive, Toronto, ON, Canada M4G $3 V 9$ \\ ${ }^{2}$ Department of Rehabilitation for the Movement Functions, Research Institute of the National Rehabilitation Center for \\ Persons with Disabilities, Tokorozawa 359-8555, Japan \\ ${ }^{3}$ Department of Medicine, University of Toronto, Toronto, ON, Canada M5T 3M6 \\ ${ }^{4}$ Department of Health \& Human Performance, University of Houston, Houston, TX 77204-6015, USA \\ ${ }^{5}$ Institute of Biomaterials and Biomedical Engineering, University of Toronto, Toronto, ON, Canada M5S 3G9
}

Correspondence should be addressed to Masae Miyatani, miyatani.masae@torontorehab.on.ca

Received 16 December 2011; Accepted 4 January 2012

Academic Editors: J. Mizrahi and C. Zwingmann

Copyright ( $(2012$ Masae Miyatani et al. This is an open access article distributed under the Creative Commons Attribution License, which permits unrestricted use, distribution, and reproduction in any medium, provided the original work is properly cited.

\begin{abstract}
Purpose. To determine the feasibility of increasing the cardiopulmonary exercise intensity during walking with gait-patterned functional electrical stimulation (GP-FES) among individuals with motor incomplete SCI. Methods. Two men with motorincomplete SCI (Subjects A and B, age 45 and 50 years; Level of Injury: C4 and T10; AIS score: D and D, resp.) performed a three sequential four-minute continuous walking sessions [(1) regular gait (non-GP-FES-1); (2) gait with GP-FES (GP-FES); (3) regular gait (non-GP-FES-2)]. Oxygen consumption $\left(V_{\mathrm{O}_{2}}\right)$ was measured continuously during trials. Results. $V \mathrm{O}_{2}$ was higher during GPFES (Subjects A and B; 14.5 and $19.1 \mathrm{~mL} / \mathrm{kg} / \mathrm{min}$, resp.) as compared to regular gait (Non-GP-FES-1: Subjects A and B; 13.4 and 17.0: $\mathrm{mL} / \mathrm{kg} / \mathrm{min}$, resp.; non-GP-FES-2: Subjects A and B; 13.1 and 17.5: $\mathrm{mL} / \mathrm{kg} / \mathrm{min}$, resp.). Conclusion. The exercise intensity of GP-FES walking was higher than that of regular walking among individuals with motor incomplete SCI. Further investigations are required to determine the clinical relevance of the exercise.
\end{abstract}

\section{Introduction}

Coronary artery disease (CAD) is the most significant complication and leading cause of mortality after spinal cord injury (SCI) [1-3]. It is most likely that the high CAD risk observed in people with SCI is related to their extreme physical inactivity [4]. Therefore, increasing physical activity may reduce CAD risk factors among people with SCI $[5,6]$. Although, in order to maximize the physiological benefits of cardiopulmonary training, exercise at an appropriate intensity is essential [7-9], the motor and autonomic impairments related to SCI often limit exercise intensity.

Several exercise interventions using functional electrical stimulation (FES), such as FES arm or leg cycle ergometry training and FES walking, have been applied to enhance physical capacity and reduce CAD risk among persons with SCI [10-12]. FES is a technology that can restore useful movements by electrically stimulating and thereby artificially contracting paralyzed or paretic muscles [13]. Applications of FES can be divided into three classes: (1) neuroprostheses for use as a permanent assistive device; (2) facilitation of exercise; (3) short term as a therapeutic intervention to improve voluntary function $[14,15]$. This latter application has been termed "FES therapy" (FET) [14].

Numerous reports over the past 30 years have asserted positive therapeutic effects of FES-assisted walking, including enhanced walking ability, among individuals with incomplete SCI [16-18]. However, there is growing evidence that regular use of FES by people with neurological impairments 


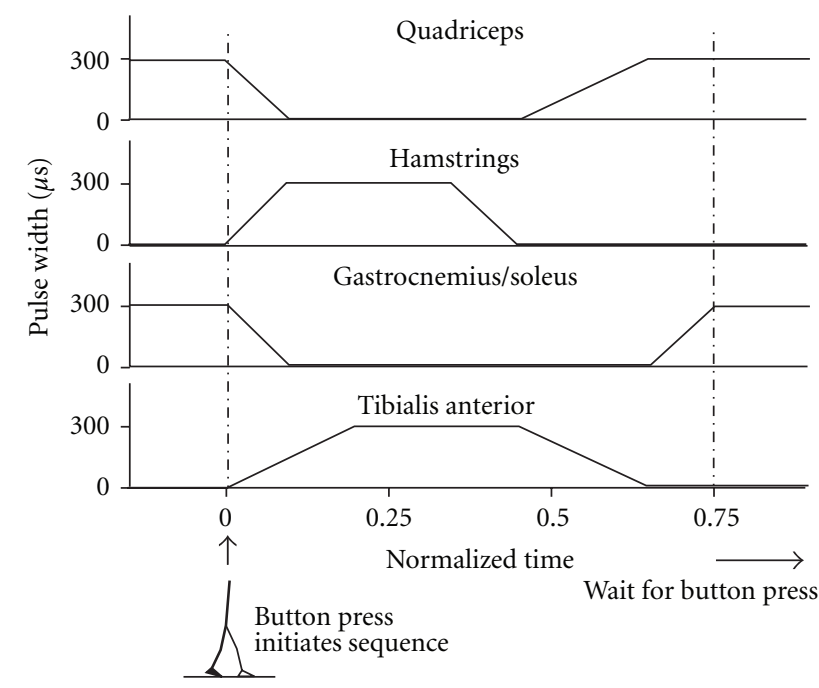

FIgURE 1: Stimulation program for a single leg. The stimulation begins from initial state representing late stance phase, and pushbutton triggers open-loop sequence beginning with swing phase.

can result in recovery of voluntary muscle control and improved functional abilities after cessation of FET [19, 20]. Many of the FES-assisted walking devices, especially those used for therapeutic interventions, induce the flexor withdrawal reflex to produce simultaneous hip flexion, knee flexion, and ankle dorsiflexion, to generate the swing phase of gait. This approach has been criticized because of the variability and rapid habituation of the flexor withdrawal reflex, and its propensity to produce nonphysiological gait patterns [18]. Thrasher et al. established a new FES system for walking termed "gait-patterned FES" (GP-FES) [18]. The stimulation patterns in GP-FES were determined based on the electromyography (EMG) recordings obtained during natural walking of able-bodied individuals (Figure 1), instead of the traditional method of inducing the flexor withdrawal reflex. They reported that following 12-16 weeks of 3 times a week GP-FES therapy, after the system is removed, the five chronic incomplete SCI individuals who took part in the study showed improved walking speeds with increases in both stride length and step frequency compared to baseline [18]. They concluded that GP-FES therapy has a potential to produce clinically relevant improvements in the gait of individuals with chronic incomplete SCI.

GP-FES stimulates both leg flexor and extensor muscles in a physiological pattern for a longer portion of the gait cycle. Therefore, it is likely that gait training with GPFES is capable of assisting individuals with SCI to increase their oxygen consumption, by increasing their leg muscle activation, as shown in prior studies describing oxygen consumption during walking with FES (but not GP-FES) [21].The purpose of this case series was to determine the feasibility of increasing the cardiopulmonary exercise intensity during walking with GP-FES among individuals with chronic incomplete SCI.

\section{Methods}

2.1. Subjects. Two adults with incomplete SCI were recruited by referral from clinical or support staff affiliated with Toronto Rehabilitation Institute, Toronto, Canada. We anticipated some acclimation effects of GP-FES on measured $\mathrm{Vo}_{2}$, thus eligible subjects for this study were people who had completed four months of the GP-FES therapy using the protocol described by Thrasher et al. [18]. Both subjects were capable of walking independently with gait aids and without supervision at baseline. Subjects able to walk independently for 12 minutes without requiring a break or manual assistance on the treadmill were eligible. Note that we had only two subjects because these conditions were rarely sufficed. The Toronto Rehabilitation Institute Research Ethics Board approved the study protocol.

2.2. Experimental Protocol. Subjects participated in the experiment during two visits, with a one week gap between the visits. During the first visit, the subjects self-selected: (a) comfortable walking speed on the treadmill during GPFES and (b) comfortable walking speed on the treadmill during regular gait (non-GP-FES). These velocities were measured and recorded for later use. Each subject wore a harness, without weight support, for safety purposes, and was permitted to hold the treadmill side rails during walking to maintain balance if needed. During the second visit, we measured oxygen consumption $\left(V_{\mathrm{o}_{2}}\right)$, while the subjects were walking with self-selected speeds measured during the first visit discussed above. During the data collection, subjects were asked to perform four tasks including: (a) 2 minutes of standing on the treadmill (REST); followed by (b) three consecutive 4 minute trials of (1) non-GP-FES-1; (2) GP-FES; (3) non-GP-FES-2 on the treadmill (Figure 2). The timeframe for data collection of 4 minutes was chosen based on the desire to limit the timeframe as much as feasible, to eliminate subject's fatigue as a confounder in the measurements and because the previously published data showed that $V_{\mathrm{o}_{2}}$ plateaus at about 2 min after the beginning of the exercise [22]. This testing protocol uses the ABA experimental design principles. The ABA experimental design is typically used to determine the effects of a treatment (in this particular case GP-FES) and the effect of removing treatment (in this particular case non-GP-FES) on the dependent variable (in this particular case of $\mathrm{VO}_{2}$ ). Thus, if there is an effect of treatment, and the effect disappears after removing the treatment, this implies an effect of treatment on the dependent variable. The other reason for implementing the ABA study design within a day was the anticipated dayto-day variations in patient performance with changes in temperature, spasticity, balance, and so forth. The ABA experimental design is generally powerful when day-to-day variability is large. A previous study demonstrated that there is larger day-to-day variability in the SCI subject's ability to ambulate, due to the effects of fatigue, balance, and spasticity on performance than same-day variability [23]. Therefore, the ABA design has been selected as the most appropriate under given circumstances. 


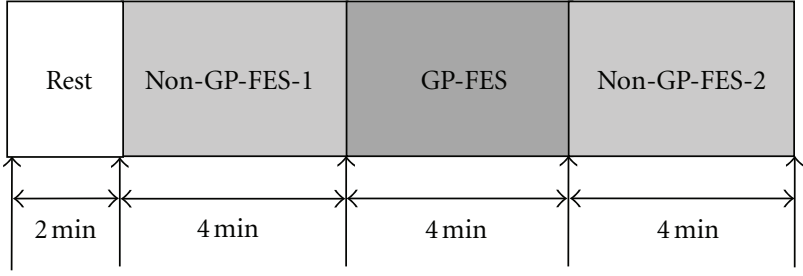

Figure 2: Experimental protocol. Rest: rest with standing position; non-GP-FES-1: first walking without GP-FES; GP-FES: walking with GP-FES; non-GP-FES-2: second walking without GP-FES.

2.3. Gait-Patterned FES (GP-FES). GP-FES has been previously described in Thrasher et al. [18]. Two portable fourchannel stimulators Compex Motion (Compex SA, Switzerland) were used to deliver the GP-FES bilaterally. Trigger buttons to initiate stimulation were connected to each stimulator. A separate set of stimulator and trigger buttons was used for each leg. Biphasic asymmetrical pulses (frequency $35 \mathrm{~Hz}$ and pulse duration $0-300 \mu \mathrm{s}$ ) were delivered to the lower extremity muscles using self-adhesive surface electrodes at four anatomic sites including the (1) quadriceps, (2) hamstrings, (3) gastrocnemius/soleus, and (4) tibialis anterior [16]. For each muscle, an investigator applied manual resistance to the appropriate joint, and gradually increased the pulse amplitude, until the subject expressed discomfort, or the investigator perceived no further increase in muscle force or change in muscle contour with further changes in pulse amplitude. Since Thrasher et al. [18] previously reported that the optimal pulse amplitude to elicit enough leg joint movements and muscle contractions to generate walking was $75 \%$ of this afore-mentioned value, pulse amplitude was set at $75 \%$ of this afore-mentioned value for each muscle group. In the GP-FES protocol, trained research staff using the two push buttons manually triggered the feed-forward FES gait pattern. Stimulation was initiated in the late stance phase of gait to initiate swing followed by stance (Figure 1). This sequence was scaled with respect to time according to the average gait cycle duration recorded during the visit one, that is, treadmill walking test completed by each subject. At the end of the sequence $(75 \%$ of the gait cycle), the stimulator would return to the initial state of extension and await the next activation of the push button.

2.4. Cardiorespiratory Measurement. During all four conditions, (REST, non-GP-FES-1, GP-FES, and non-GP-FES-2) $V_{\mathrm{O}_{2}}(\mathrm{~mL} / \mathrm{kg} / \mathrm{min})$ was recorded using a telemetric breath-bybreath respiration measurement system K4b2 (Cosmed Inc, Italy). Pulmonary ventilation (VE) and heart rate (HR) were also recorded. Data were continuously acquired and analyzed in real time. After the data collections, each cardiorespiratory parameter was averaged during the last 1 minute of resting stage (Subjects A and B; 12 and 21 data points, resp.) and during the last 2 minutes of each walking stage (Subjects A and B; 37-40 and 64-76 data points, resp.).

To determine the exercise intensity associated with each task, metabolic equivalents (METs) were calculated as an index of exercise intensity for all four conditions. METs
TABle 1: Demographic and impairment characteristics of subjects.

\begin{tabular}{lcc}
\hline Subject & Subject A & Subject B \\
\hline Age (years) & 45 & 50 \\
Sex & $\mathrm{M}$ & $\mathrm{M}$ \\
Height $(\mathrm{cm})$ & 175 & 170.2 \\
Weight $(\mathrm{kg})$ & 77 & 83.1 \\
Neurologic level & $\mathrm{C} 4$ & $\mathrm{~T} 10$ \\
AIS & $\mathrm{D}$ & $\mathrm{D}$ \\
Year of postinjury & 3 & 34 \\
\hline
\end{tabular}

values are the fraction of the $V_{\mathrm{O}_{2}}$ during motor tasks relative to the resting $\mathrm{Vo}_{2}$ :

$$
\text { METs }=\frac{V_{\mathrm{o}_{2}} \text { During gait }(\mathrm{mL} / \mathrm{kg} / \mathrm{min})}{\text { Resting } V \mathrm{o}_{2}(\mathrm{~mL} / \mathrm{kg} / \mathrm{min})},
$$

where one MET is equivalent to the resting metabolic rate of $2.7 \mathrm{mml} / \mathrm{kg} / \mathrm{min}[24]$.

\section{Results}

Table 1 summarizes the subjects' demographic and impairment characteristics. Both subjects were able to perform the four tasks without difficulty, exhaustion or stumbling. Table 2 shows the mean (SD) gait speed, $V_{\mathrm{O}_{2}}, \mathrm{VE}, \mathrm{HR}$, and MET values for each subject during each phase of visit two. The comfortable walking speeds of subjects were the same during GP-FES and non-GP-FES. In both subjects, $\mathrm{Vo}_{2}$ increased during GP-FES compared to both non-GP-FES-1 and non-GP-FES-2. However, VE and HR tended to increase through non-GP-FES-1, GP-FES and non-GP-FES-2. METs values were 5.0 and 6.3 METs during non-GP-FES-1, 4.9 and 6.5 METS during non-GP-FES-2, and 5.4 and 7.1 METs during GP-FES in subject A and subject B, respectively.

\section{Discussion}

The study findings indicate that the exercise intensity during GP-FES tends to be larger than that of walking without GPFES. The observed change during GP-FES in $\mathrm{VO}_{2}$ is probably accounted for by the recruitment of additional muscle fibres through GP-FES, which the subjects cannot voluntarily activate themselves due to their spinal cord impairment.

The following is the likely explanation for the observed findings. We assume that the increment of $\mathrm{Vo}_{2}$ was mainly due to GP-FES because GP-FES facilitates additional leg muscle fibre recruitment above and beyond the muscle fibres activation that normally occurred during voluntarily executed locomotion. As a result, we were able to observe increase in oxygen utilization during GP-FES as compared to two non-GP-FES conditions. However, $V_{\mathrm{O}_{2}}$ is usually determined not only by examining the oxygen utilization in active muscles, but also by observing the VE [25]. In our experiments, the VE tended to increase throughout the sessions. Therefore, we were not able to assert that the increase in $\mathrm{Vo}_{2}$ during GP-FES could be solely attributed 
TABLE 2: Walking speed, oxygen consumption $\left(V_{\mathrm{O}_{2}}\right)$, Pulmonary ventilation (VE), heart rate (HR), and METs values during non-GP-FES and GP-FES walking, where rest is rest with standing position; non-GP-FES-1 is first walking without GP-FES; GP-FES is walking with GPFES; non-GP-FES-2 is second walking without GP-FES. Each cardiorespiratory parameter was averaged during the last 1 minute of resting stage and during the last 2 minutes of each walking stage.

\begin{tabular}{|c|c|c|c|c|}
\hline Subject & Rest & Non-GP-FES-1 & GP-FES & Non-Gp-FES-2 \\
\hline \multicolumn{5}{|c|}{ Walking speed $(\mathrm{m} / \mathrm{min})$} \\
\hline Subject A & - & 35 & 35 & 35 \\
\hline Subject B & - & 18.3 & 18.3 & 18.3 \\
\hline \multicolumn{5}{|c|}{$V_{\mathrm{O}_{2}}(\mathrm{~mL} / \mathrm{min} / \mathrm{kg})$} \\
\hline Subject A & 3.6 & 13.4 & 14.5 & 13.1 \\
\hline Subject B & 4.9 & 17.0 & 19.1 & 17.5 \\
\hline \multicolumn{5}{|c|}{$\mathrm{VE}(\mathrm{mL} / \mathrm{min} / \mathrm{kg})$} \\
\hline Subject A & 11.8 & 26.7 & 32.8 & 32.2 \\
\hline Subject B & 11.9 & 31.2 & 37.0 & 38.1 \\
\hline \multicolumn{5}{|c|}{ HR (beat/min) } \\
\hline Subject A & 69.3 & 94.8 & 98.9 & 100.7 \\
\hline Subject B & 82.0 & 113.7 & 128.0 & 141.0 \\
\hline \multicolumn{5}{|l|}{ METs } \\
\hline Subject A & 1.3 & 5.0 & 5.4 & 4.9 \\
\hline Subject B & 1.8 & 6.3 & 7.1 & 6.5 \\
\hline
\end{tabular}

to the changes due to the additional, FES-induced, muscle activation. However, if the increment of $\mathrm{VO}_{2}$ was only due to increased $\mathrm{VE}, \mathrm{VE}$, and $\mathrm{Vo}_{2}$ should show similar trends. But in this study, VE kept increasing through all sessions, while $\mathrm{Vo}_{2}$ only increased during GP-FES, as compared to non-GP-FES-1 and non-GP-FES-2 conditions. This implies that the increase in $V_{\mathrm{O}_{2}}$ can be attributed not only to the $\mathrm{VE}$, but also to the increased oxygen utilization in the exercising muscles. Another potential factor affecting $\mathrm{Vo}_{2}$ during GPFES is arm usage as the patients kept their body balance using treadmill frames [26]. However, we believe that the increase in $V_{\mathrm{O}_{2}}$ during GP-FES was mainly due to the FES since the patients had relatively high functions and did not require much support from the treadmill frame. Further, in a previous study, it was shown that the application of FES to the lower limbs reduced the muscle activity in the upper arms instead of increasing them [27], which partially supports our assumption.

There are two previous studies, which determined oxygen consumption during walking with FES in people with incomplete SCI, while they did not use GP-FES. Stein et al. (1993) investigated energy consumption during walking with FES (1-4 channels with either surface, percutaneous or implanted electrodes) [28]. On average, the speed of locomotion was higher during walking with FES as compared to walking without FES, while the oxygen consumption was reduced somewhat. Sykes et al. investigated energy consumption and energy cost during walking, using the reciprocating gait orthosis (RGO) with FES (surface stimulation of quadriceps and hamstrings) [21]. In their study, walking speed and energy cost remained unchanged in one subject, whereas another subject increased his walking speed by $14.0 \%$, increased his energy consumption by $8.4 \%$, and his energy cost remained unchanged. Three subjects increased their walking speeds (by 12.4, 12.7, and 6.8\%), energy consumption (by 25.9, 20.4, and $18.4 \%$ resp.) and energy cost (by 11.6, 6.8, and 10.5\%, resp.). On average, the energy consumption was increased by $15.3 \%$ during RGO walking with FES as compared to RGO walking without FES assistance. There results were contradictory in the sense that the additional FES increases the oxygen consumption or not, while the walking conditions, that is, with orthosis or not, and stimulated muscles were different. Our experiment was also in a different condition, that is, waling on a treadmill and with GP-FES. Therefore, we cannot compare our result and their results directly. However, we can conclude that the GP-FES walking has a potential to increase the oxygen consumption during walking and that this increase in oxygen consumption can be contributed to the increase in oxygen requirement of the stimulated muscles during FES-assisted walking.

Although the absolute increase in the exercise intensity from 1st non-GP-FES to GP-FES was small ( 0.4 and $0.8 \mathrm{METs})$, the relative increments were considerable (8.5, $12.7 \%$, resp.). This suggests that the current result was not in the range of measurement error. The absolute METs values during GP-FES were 5.4 and 7.1 METs (Table 2). Based on most international physical activity guidelines for able-bodied people and currently published physical activity guideline for people with SCI, a moderate to vigorous intensity exercise, which is 3.0-6.0 METs, to maintain or improve health, including CAD risk are recommended [79]. In this sense, the obtained METs during GP-FES implied that the exercise intensity of GP-FES met the recommended exercise intensity. However, as the METs values without GPFES were already within this recommended value range due to the patients' fitness levels, the clinical relevance of these increments to GP-FES remains unclear. Further investigation is needed to determine the clinical relevance of this increment to exercise intensity through intervention 
studies using the GP-FES. Also more investigation is required for the change of HR, since HR kept increasing through all sessions, although we anticipated $\mathrm{HR}$ to show same characters as $\mathrm{Vo}_{2}$.

In conclusion, we demonstrated the feasibility of increasing the exercise intensity during walking through use of GP-FES walking among individuals with chronic incomplete SCI. Further investigations are required to determine the clinical relevance of increases in $V_{\mathrm{O}_{2}}$ through a GP-FES training intervention study aiming at preventing CAD or reducing CAD-related morbidity among individuals with SCI. With the current results, we propose future longitudinal studies with a larger sample of patients' with diverse walking ability.

\section{Acknowledgments}

The authors are particularly grateful to Dr. Tom Chau, Bloorview Kids Rehab, for his support in the experiment and to Dr. Minoru Shinohara, Georgia Institute Technology for his help with the paper. We also thank Mr. Albert Vett and Mr. Milad Alizadeh-Meghrazi for their support. The author (M. Miyatani) is a fellow funded by Canadian Paraplegic Association Ontario and Ontario Neurotrauma Foundation. This research was supported by funding from the Ontario Neurotrauma Foundation Grant 2004-SCI-SC-04.

\section{References}

[1] R. Banerjea, U. Sambamoorthi, F. Weaver, M. Maney, L. M. Pogach, and T. Findley, "Risk of stroke, heart attack, and diabetes complications among veterans with spinal cord injury," Archives of Physical Medicine and Rehabilitation, vol. 89, no. 8, pp. 1448-1453, 2008.

[2] E. Garshick, A. Kelley, S. A. Cohen et al., "A prospective assessment of mortality in chronic spinal cord injury," Spinal Cord, vol. 43, no. 7, pp. 408-416, 2005.

[3] S. H. Orakzai, R. H. Orakzai, N. Ahmadi et al., "Measurement of coronary artery calcification by electron beam computerized tomography in persons with chronic spinal cord injury: evidence for increased atherosclerotic burden," Spinal Cord, vol. 45, no. 12, pp. 775-779, 2007.

[4] K. A. Martin Ginis, A. E. Latimer, K. P. Arbour-Nicitopoulos et al., "Leisure time physical activity in a population-based sample of people with spinal cord injury part I: demographic and injury-related correlates," Archives of Physical Medicine and Rehabilitation, vol. 91, no. 5, pp. 722-728, 2010.

[5] P. J. Manns, J. A. McCubbin, and D. P. Williams, "Fitness, inflammation, and the metabolic syndrome in men with paraplegia," Archives of Physical Medicine and Rehabilitation, vol. 86, no. 6, pp. 1176-1181, 2005.

[6] A. C. Buchholz, K. A. Martin Ginis, S. R. Bray et al., "Greater daily leisure time physical activity is associated with lower chronic disease risk in adults with spinal cord injury," Applied Physiology, Nutrition and Metabolism, vol. 34, no. 4, pp. 640$647,2009$.

[7] D. E. Warburton, P. T. Katzmarzyk, R. E. Rhodes, and R. J. Shephard, "Evidence-informed physical activity guidelines for Canadian adults," Canadian Journal of Public Health, vol. 98, supplement 2, pp. S16-S68, 2007.
[8] A. L. Hicks, K. A. Martin Ginis, C. A. Pelletier, D. S. Ditor, B. Foulon, and D. L. Wolfe, "The effects of exercise training on physical capacity, strength, body composition and functional performance among adults with spinal cord injury: a systematic review," Spinal Cord, vol. 49, no. 11, pp. 1103-1127, 2011.

[9] K. A. Martin Ginis, A. L. Hicks, A. E. Latimer et al., "The development of evidence-informed physical activity guidelines for adults with spinal cord injury," Spinal Cord, vol. 49, no. 11, pp. 1088-1096, 2011.

[10] P. L. Jacobs, M. S. Nash, K. J. Klose, R. S. Guest, B. M. Needham-Shropshire, and B. A. Green, "Evaluation of a training program for persons with SCI paraplegia using the Parastep1 ambulation system: part 2. Effects on physiological responses to peak arm ergometry," Archives of Physical Medicine and Rehabilitation, vol. 78, no. 8, pp. 794-798, 1997.

[11] T. Mohr, J. L. Andersen, F. Biering-Sørensen et al., "Long term adaptation to electrically induced cycle training in severe spinal cord injured individuals," Spinal Cord, vol. 35, no. 1, pp. 1-16, 1997.

[12] D. H. J. Thijssen, P. Heesterbeek, D. J. M. van Kuppevelt, J. Duysens, and M. T. E. Hopman, "Local vascular adaptations after hybrid training in spinal cord-injured subjects," Medicine and Science in Sports and Exercise, vol. 37, no. 7, pp. 11121118, 2005.

[13] M. R. Popovic, A. Curt, T. Keller, and V. Dietz, "Functional electrical stimulation for grasping and walking: indications and limitations," Spinal Cord, vol. 39, no. 8, pp. 403-412, 2001.

[14] L. R. Sheffler and J. Chae, "Neuromuscular electrical stimulation in neurorehabilitation," Muscle and Nerve, vol. 35, no. 5, pp. 562-590, 2007.

[15] E. J. Nightingale, J. Raymond, J. W. Middleton, J. Crosbie, and G. M. Davis, "Benefits of FES gait in a spinal cord injured population," Spinal Cord, vol. 45, no. 10, pp. 646-657, 2007.

[16] M. R. Popovic, T. Keller, I. P. I. Pappas, V. Dietz, and M. Morari, "Surface-stimulation technology for grasping and walking neuroprostheses: improving quality of life in stroke/ spinal cord injury subjects with rapid prototyping and portable FES systems," IEEE Engineering in Medicine and Biology Magazine, vol. 20, no. 1, pp. 82-93, 2001.

[17] A. C. B. Smith, G. F. Phillips, and B. J. Andrews, "An exercise regime using Electrical Stimulation in rehabilitation program for incomplete spinal cord injured patients," in Proceedings of the 25th Anniversary Strathclyde Bioengineering Unit, Glasgow, Scotland, 1988.

[18] T. A. Thrasher, H. M. Flett, and M. R. Popovic, "Gait training regimen for incomplete spinal cord injury using functional electrical stimulation," Spinal Cord, vol. 44, no. 6, pp. 357-361, 2006.

[19] T. Bajd, A. Kralj, M. Štefančič, and N. Lavrač, "Use of functional electrical stimulation in the lower extremities of incomplete spinal cord injured patients," Artificial Organs, vol. 23, no. 5, pp. 403-409, 1999.

[20] N. Donaldson, T. A. Perkins, R. Fitzwater, D. E. Wood, and F. Middleton, "FES cycling may promote recovery of leg function after incomplete spinal cord injury," Spinal Cord, vol. 38, no. 11, pp. 680-682, 2000.

[21] L. Sykes, I. G. Campbell, E. S. Powell, E. R. S. Ross, and J. Edwards, "Energy expenditure of walking for adult patients with spinal cord lesions using the reciprocating gait orthosis and functional electrical stimulation," Spinal Cord, vol. 34, no. 11, pp. 659-665, 1996.

[22] R. B. Stein, S. L. Chong, K. B. James, and G. J. Bell, "Improved efficiency with a wheelchair propelled by the legs using 
voluntary activity or electric stimulation," Archives of Physical Medicine and Rehabilitation, vol. 82, no. 9, pp. 1198-1203, 2001.

[23] R. J. Marino, G. Scivoletto, M. Patrick et al., "Walking index for spinal cord injury version 2 (WISCI-II) with repeatability of the 10-m walk time: inter- and intrarater reliabilities," American Journal of Physical Medicine and Rehabilitation, vol. 89, no. 1, pp. 7-15, 2010.

[24] E. G. Collins, D. Gater, J. Kiratli, J. Butler, K. Hanson, and W. E. Langbein, "Energy cost of physical activities in persons with spinal cord injury," Medicine and Science in Sports and Exercise, vol. 42, no. 4, pp. 691-700, 2010.

[25] J. H. Wilmore and D. L. Costill, "Adequacy of the Haldane transformation in the computation of exercise $\mathrm{V}\left(\mathrm{O}_{2}\right)$ in man," Journal of Applied Physiology, vol. 35, no. 1, pp. 85-89, 1973.

[26] E. Isakov, J. Mizrahi, D. Graupe, E. Becker, and T. Najenson, "Energy cost and physiological reactions to effort during activation of paraplegics by functional electrical stimulation," Scandinavian Journal of Rehabilitation Medicine, vol. 17, no. 12, pp. 102-107, 1985.

[27] J. S. Petrofsky and J. B. Smith, "Physiologic costs of computercontrolled walking in persons with paraplegia using a reciprocating-gait orthosis," Archives of Physical Medicine and Rehabilitation, vol. 72, no. 11, pp. 890-896, 1991.

[28] R. B. Stein, M. Belanger, G. Wheeler et al., "Electrical systems for improving locomotion after incomplete spinal cord injury: an assessment," Archives of Physical Medicine and Rehabilitation, vol. 74, no. 9, pp. 954-959, 1993. 


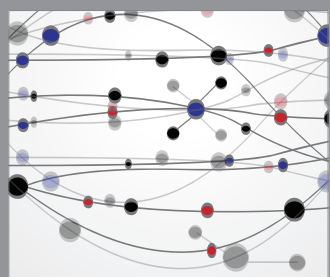

The Scientific World Journal
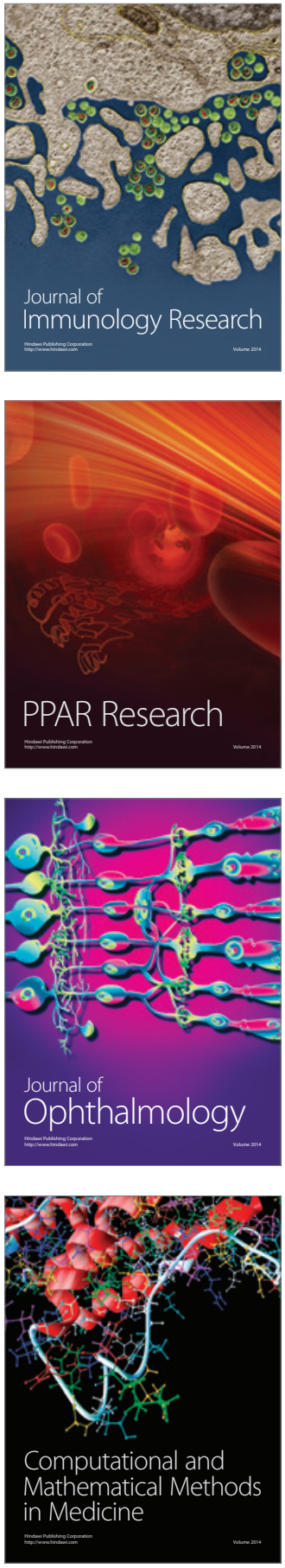

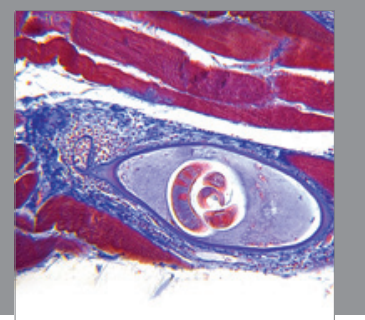

Gastroenterology

Research and Practice
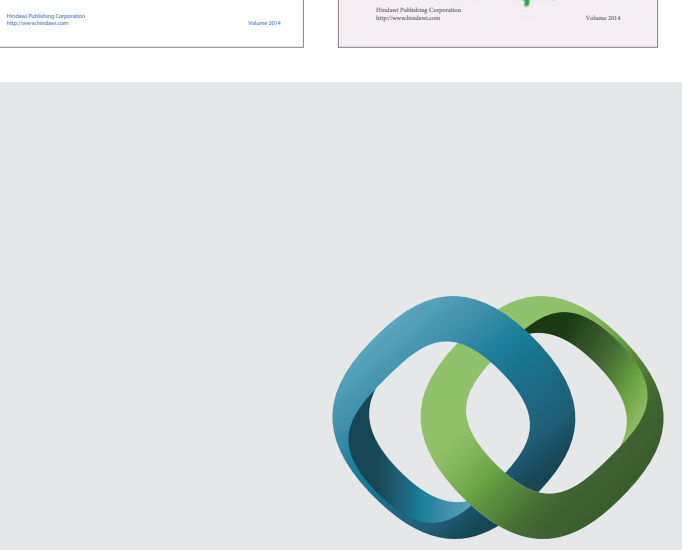

\section{Hindawi}

Submit your manuscripts at

http://www.hindawi.com
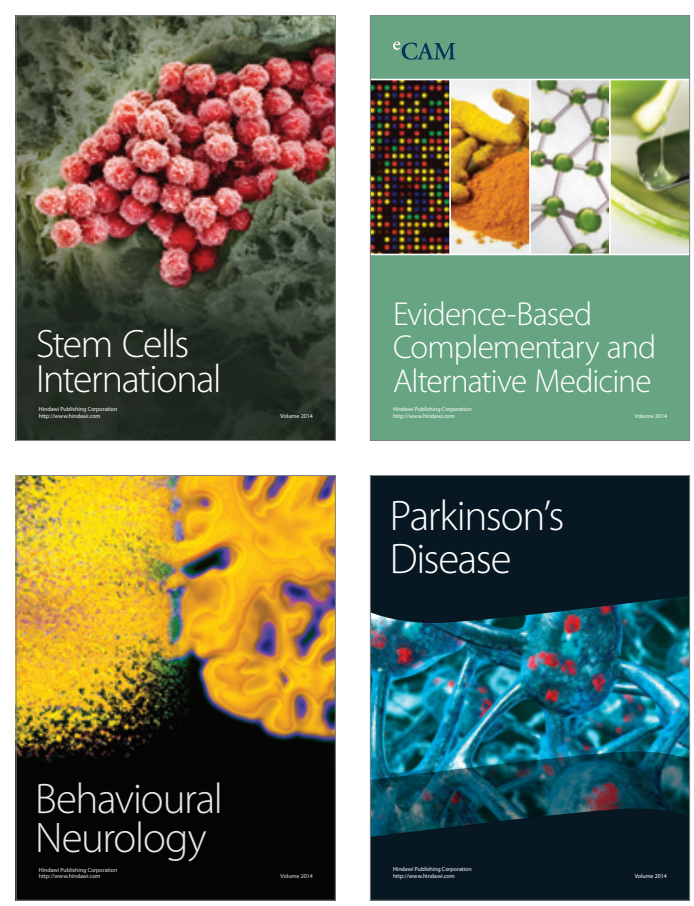

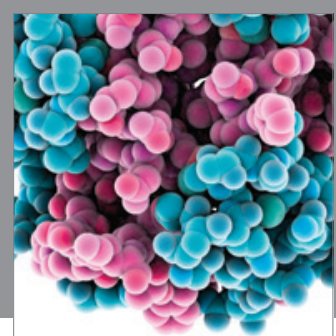

Journal of
Diabetes Research

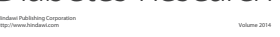

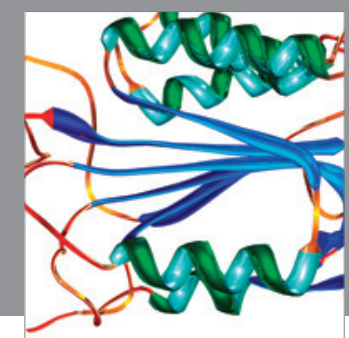

Disease Markers
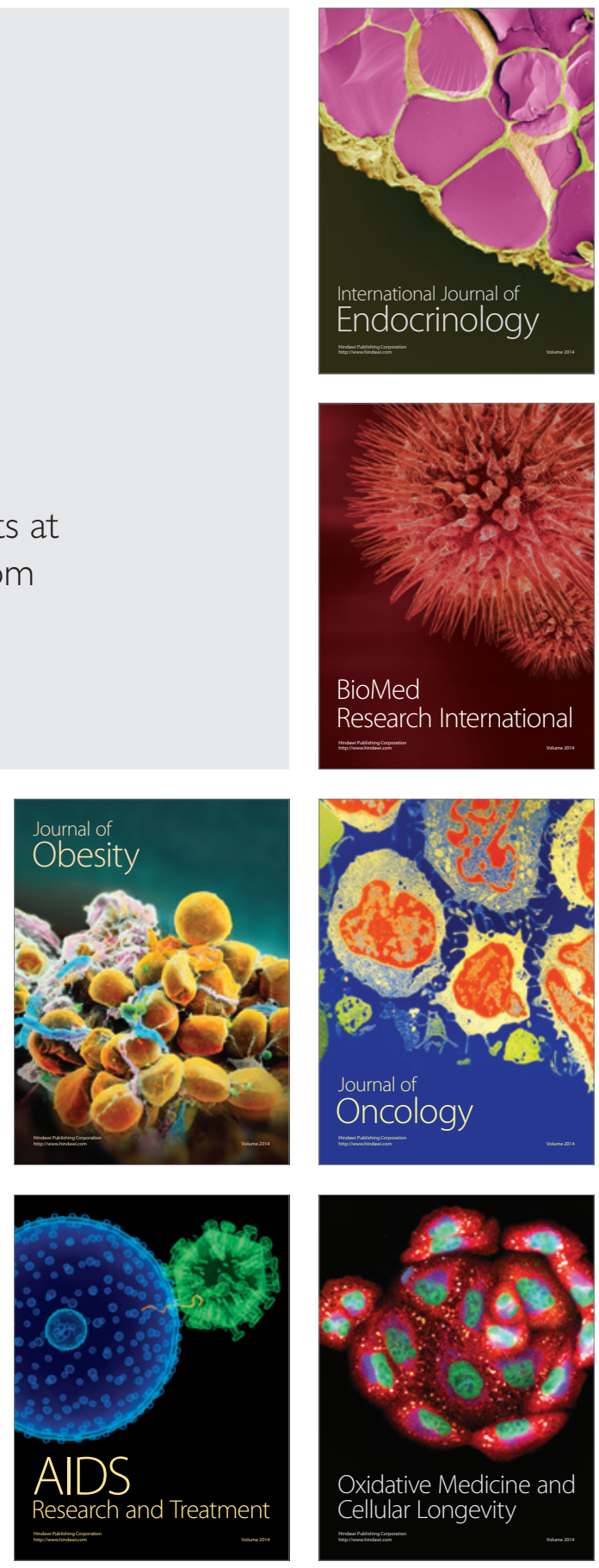